\title{
Brief 8: Grete Hermann an ihre Mutter [Auguste Hermann (geb. Leipoldt)] über ihre Gespräche mit Heisenberg in Leipzig und andere philosophische Arbeitsberichte $(5.3 .1934)^{1}$
}

\section{III. 34}

Hier ist das Semester zu Ende, Heisenberg ist für einige Wochen fort. Die Diskussionen mit ihm sind also erst mal abgebrochen. In der vorigen Woche haben wir sie noch intensiv geführt, von beiden Seiten mit der Absicht, einen gewissen inhaltlichen Abschluss zu erzwingen. Einigermaßen ist das auch gelungen, wenn wir auch nicht einig geworden sind über das ganze Kausalproblem, so doch anscheinend über die physikalischen Fragen, die damit zusammenhängen. Der Gegensatz hat sich ins philosophische Gebiet verschoben, wo ich nun weiter anzupacken gedenke. Ich bleibe vorläufig hier, um das bisherige Ergebnis in Ruhe zu verarbeiten, und, wenn's geht, nach Heisenberg's Rückkehr noch einmal mit ihm durchzusprechen.

1 Der Brief beginnt hier mit „So [berichtete] (Anm. Hrsg.: Wort wieder gestrichen) Grete an unsere Mutter über ihre Gespräche mit Heisenberg. U. andere philosophische Arbeitsberichte." Der Verfasser ist vermutlich Grete Henry-Hermanns Bruder Carl Heinrich Hermann (Physiker, Kristallograph, geb. 17.6.1898 in Wesermünde-Lehe, gest. 12.9.1961, Marburg/Lahn).

(C) Springer Fachmedien Wiesbaden GmbH, ein Teil von Springer Nature 2019

K. Herrmann (Hrsg.), Grete Henry-Hermann: Philosophie - Mathematik-

Quantenmechanik, Frauen in Philosophie und Wissenschaft. Women

Philosophers and Scientists, https://doi.org/10.1007/978-3-658-16241-2_30 